\title{
Effects of aging on interference control in selective attention and working memory
}

\author{
Selene Cansino • Daniela Guzzon • \\ Massimiliano Martinelli • Michele Barollo • Clara Casco
}

Published online: 10 May 2011

(C) Psychonomic Society, Inc. 2011

\begin{abstract}
Working memory decay in advanced age has been attributed to a concurrent decrease in the ability to control interference. The present study contrasted a form of interference control in selective attention that acts upon the perception of external stimuli (access) with another form that operates on internal representations in working memory (deletion), in order to determine both of their effects on working memory efficiency in younger and older adults. Additionally, we compared memory performance under these access and deletion functions to performance in their respective control conditions. The results indicated that memory accuracy improved in both age groups from the access functions, but that only young adults benefited from the deletion functions. In addition, intrusion effects in the deletion condition were larger in older than in younger adults. The ability to control the irrelevant perception- and memory-elicited interference did not decline in general with advancing age; rather, the control mechanisms that operate on internal memory representations declined specifically.
\end{abstract}

Keywords Aging - Working memory - Selective attention · Interference control $\cdot$ Inhibition

Several cognitive functions depend on the ability to control interference from irrelevant external and internal stimuli that

\footnotetext{
S. Cansino $(\bowtie)$

Laboratorio de NeuroCognición, Facultad de Psicología, Universidad Nacional Autónoma de México,

Avenida Universidad 3004, Colonia Copilco Universidad, México, DF 04510, México

e-mail: selene@unam.mx

D. Guzzon $\cdot$ M. Martinelli $\cdot$ M. Barollo $\cdot$ C. Casco

Dipartimento di Psicologia Generale,

Università degli Studi di Padova,

Padova, Italia
}

hamper the processing of information that is relevant for the task at hand. Most researchers agree that several top-down cognitive processes that depend on the prefrontal cortex control interference (see, e.g., Miller \& Cohen, 2001; Nee \& Jonides, 2009; West, 1996); however, there is little agreement on the specific mechanisms involved in resolving interference. Some models have proposed that inhibition is responsible for controlling interference (Anderson \& Bjork, 1994; Clark, 1996; Hasher \& Zacks, 1988; MacLeod, 2007). According to Hasher and Zacks, familiar stimuli initiate representations automatically, activating irrelevant information that must be suppressed or regulated via inhibition mechanisms. Other authors have proposed that accurate retrieval cues and source-monitoring processes that enhance attention toward relevant information control interference (Unsworth \& Engle, 2007). The active maintenance of relevant information for goal-directed behavior is another mechanism that might control interference (Braver, Gray, \& Burgess, 2007; Kane, Conway, Hambrick, \& Engle, 2007).

Irrelevant external stimuli, or distractors, activate cognitive control functions in the perceptual or selective attention stages of processing, but their behavioral effects are manifested in the subsequent task for which selective attention functions are initiated. Alternatively, the interference from internal irrelevant representations activates cognitive control mechanisms in subsequent stages of processing such as working memory. Thus, memory-based internal interference occurs concurrently with working memory. The inability to control distracting percepts and memories affects working memory efficiency, because when irrelevant information is accepted into working memory and maintained, it increases competition for the limited resources or capacity of the working memory system (Conway \& Engle, 1994). This competition is considered a primary cause of forgetting information (Hasher \& Zacks, 1988). 
In the framework proposed by Hasher, Zacks, and May (1999), interference is controlled by three inhibition-related processes: access, deletion, and restraint. Restraint stops prepotent thoughts or behavioral responses, and access and deletion operate on the information processed by different cognitive functions. Access is the inhibition function responsible for ignoring concurrent distractions, whereas deletion stops the processing of information that is no longer relevant. The conceptual distinction between access and deletion functions corresponds to the interference control mechanisms that operate in selective attention and working memory, respectively, as described above. Therefore, the terms "access" and "deletion" will be used in the present study to refer to these two distinct mechanisms. However, these terms should not be taken to indicate that interference is exclusively regulated by means of inhibition functions in the present study. Several cognitive control functions, in addition to inhibition control, may be responsible for preventing irrelevant information from entering working memory and for controlling interference from irrelevant memory representations.

Although several researchers agree that different cognitive control functions operate on selective attention and working memory to circumvent interference (Hasher et al., 1999; Nigg, 2000; Wilson \& Kipp, 1998), few studies (Dumas \& Hartman, 2008; Nee \& Jonides, 2008, 2009; Palladino, Mammarella, \& Vecchi, 2003) have examined these interference control functions within the same subjects and under equivalent conditions. With the exception of one study (Dumas \& Hartman, 2008), the agerelated effects of access and deletion on working memory performance have always been evaluated in separate experiments. In this study, the access and deletion functions were evaluated, respectively, by displaying a cue along with the stimuli and after stimulus presentation, to indicate which stimuli were relevant and which were irrelevant for the working memory task that followed. Dumas and Hartman found that, in both conditions, the accuracy in remembering words and the intrusion error rates were equivalent in young and older adults. Intrusion errors in this study were defined as the number of irrelevant words selected in the test.

When these interference control mechanisms have been evaluated independently, the results have been characterized by inconsistency, which may be attributed to the different procedures that have been used to assess these mechanisms. The access function is evaluated through tasks that allow the experimenters to manipulate the activation level of irrelevant information, such as the Stroop task or the AX continuous performance test (Rush, Barch, \& Braver, 2006). These procedures require a combination of interference control functions that act on external percepts and on prepotent responses, which complicates the evalu- ation of how efficiently each of these control mechanisms is applied by the subjects. Several researchers (e.g., Cohn, Dustman, \& Bradford, 1984; Comalli, Wapner, \& Werner, 1962; Hartman \& Hasher, 1991; Houx, Jolles, \& Vreeling, 1993) have reported less effective interference control mechanisms in the elderly with the Stroop task. However, Verhaeghen and Meersman (1998) provided a meta-analysis that contradicts these findings. By using the AX continuous performance test, which evaluates subjects' ability to control a prepotent response tendency induced by context information, Rush et al. (2006) provided no evidence of interference control failing in older adults.

The negative-priming paradigm (Tipper, 1985) has also been used to evaluate access functions. Negative priming is the additional time required to respond to a target trial whose stimulus was presented in the previous trial, relative to a control condition, whose stimulus is different from the one in the previous trial. An alternative interpretation is that this response delay is due to the retrieval of a memory trace that conflicts with the current trial (Neill \& Valdes, 1992). The absence of negative priming in older adults relative to young subjects (see, e.g., Hasher, Stoltzfus, Zacks, \& Rypma, 1991; Kane, Hasher, Stoltzfus, Zacks, \& Connelly, 1994; McDowd \& Filion, 1995; Stoltzfus, Hasher, Zacks, Ulivi, \& Goldstein, 1993) has been interpreted as evidence that older adults fail to activate inhibition processes against the irrelevant stimulus from the priming trial. However, negative priming is not always absent in older adults (e.g., Gamboz, Russo, \& Fox, 2002; Kieley \& Hartley, 1997; Kramer, Humphrey, Larish, \& Logan, 1994; Sullivan \& Faust, 1993). Access mechanisms in aging may also be studied by evaluating the subject's ability to perform a task under distraction conditions. Visual searching (e.g., Plude \& Doussard-Roosevelt, 1989; Scialfa, Esau, \& Joffe, 1998) and reading comprehension (e.g., Carlson, Hasher, Connelly, \& Zacks, 1995; Connelly, Hasher, \& Zacks, 1991) abilities are especially vulnerable in older adults when performance is assessed under distractor conditions.

The deletion function and its relationship to age-related working memory decline have been studied using the reading span test (Daneman \& Carpenter, 1980). In this procedure, subjects are instructed to remember the final words from sentences presented in sets, while the number of sentences gradually increases. The analysis of intrusion errors in this task, computed as the number of nonfinal words that the subjects recall, provides a direct and objective measurement of interference control abilities. Using this task, some researchers have found significantly more intrusion errors in older than in young adults (Robert, Borella, Fagot, Lecerf, \& Ribaupierre, 2009), while other researchers have not (Schelstraete \& Hupet, 2002). However, variations in the reading span test have demonstrated that an interference control failure might account for poor 
performance on this task, but so also might proactive interference from previous trials (Lustig, May, \& Hasher, 2001; May, Hasher, \& Kane, 1999), the length of the reading span task (Robert et al., 2009), or the subjects' reading skills (Chiappe, Hasher, \& Siegel, 2000; De Beni, Palladino, Pazzaglia, \& Cornoldi, 1998).

In the visuospatial domain, the deletion function was evaluated using a task similar to the reading span test. Cornoldi, Bassani, Berto, and Mammarella (2007) presented sequences of $4 \times 4$ blank matrices to their subjects while the experimenter pointed randomly to three positions in each matrix. Subjects were asked to remember only the final position signaled in each matrix. Intrusion errors were computed as the number of nonfinal positions the subjects recalled. Young and elderly subjects both produced more intrusion errors than invention errors, which were identifications of positions never signaled by the experimenter.

Researchers have also studied the deletion function in working memory by displaying a cue after presenting a set of stimuli (Andrés, Van der Linden, \& Parmentier, 2004; Oberauer, 2001, 2005b) to indicate which stimuli are task relevant. Those that are irrelevant should then be unselected or inhibited. This procedure provides a direct measure of interference control via the failure to detect intrusion probes (i.e., stimuli that have been cued as irrelevant). This method has been tested using both letter trigrams (Andrés et al., 2004) and words (Oberauer, 2001, 2005b) as stimuli. Two of these studies found significantly larger intrusion costs in older than in younger adults with regard to reaction times (Oberauer, 2001, Exp. 1; 2005b, Exp. 1). Andrés et al. calculated intrusion errors as the numbers of letters recalled from the irrelevant trigram and found that this type of error occurred at significantly higher rates in elderly subjects. Furthermore, intrusion error rates in older adults were significantly higher than in younger adults in a working memory task that required subjects to simultaneously update relevant information and inhibit or deselect irrelevant information (De Beni \& Palladino, 2004). De Beni and Palladino required subjects to remember the three smallest items from a list of 10 words (Exp. 1) or 12 words (Exp. 2).

Previous studies on the access and deletion functions have yielded conflicting results. The existing evidence is insufficient to determine whether older adults have a reduced capacity to filter out or avoid irrelevant information while their working memory is tested. There have been several attempts to explain these inconsistent results. One explanation of the discrepancies is that the results from several interference control tasks can also be attributed to source memory failure (i.e., remembering the temporal and spatial context in which personal experiences take place), which decreases with advancing age (Cansino, 2009). In the case of the reading span test and certain visuospatial tasks (Cornoldi et al., 2007), only the final word of each sentence or the final position must be remembered; thus, the contextual information of the relevant stimulus must also be encoded for subjects to succeed in these tasks (Lustig et al., 2001). An explanation based on memory rather than the failure to control interference might also explain the working memory task performance of subjects (Oberauer, 2005a, Exp. 2).

The question to be determined in the present study was whether the access and deletion functions differ between age groups and to measure the extent to which these mechanisms influence working memory efficiency within each age group. Given the difficulties outlined above, we evaluated access and deletion functions in the same subjects under the same conditions. Moreover, we compared each interference control function to its respective control condition, which required memory processes but not interference control mechanisms. This comparison allowed us to determine the specific effect of each type of interference control mechanism on memory performance.

The study was designed to evaluate the access and deletion functions in the visuospatial domain using Gabors (sinusoidal luminance signals within a Gaussian envelope) as stimuli. Gabors provide stimuli largely protected from cultural or verbal influences. Moreover, because a Gabor contrast was used that was higher than the contrast threshold obtained at the same spatial frequency in the contrast sensitivity function, the measured effects on working memory were unaffected by the reduced contrast sensitivity in elderly subjects.

The present study introduces a novel working memory paradigm that builds on tasks that evaluate intrusion error rates as a direct measure of interference control efficiency. The task consisted of sequentially presenting two circles composed of Gabor elements (Fig. 1), in which one, two, or three Gabors were missing (one-, two-, and three-gap stimuli). Then, the probe was presented with only one missing Gabor. In each trial, subjects judged whether the position of the gap presented in the probe was also a gap in one of the two test stimuli. To indicate which of the two test stimuli was relevant in each trial, a cue made of two small circles, one empty and one filled, was presented. The filled circle signaled whether the first or the second stimulus was relevant. In the access condition, the cue was presented before the test stimuli, and in the deletion condition, after. The gap in the probe could match one of the gaps of the relevant stimulus (positive probe), of the irrelevant stimulus (intrusion probe), or of neither of them (negative probe). An intrusion error occurred when subjects judged that the gap position in the probe was one of the gap positions from the relevant stimulus, but instead it was one of the gap positions from the irrelevant stimulus. However, to truly examine intrusion effects without the influence of a possible bias in the response criterion, intrusion error rates 
Fig. 1 The working memory paradigm employed in the access and deletion conditions, as well as their respective control conditions. The only difference between the inhibition and control conditions is that we used two empty circles as a cue in the control conditions, whereas we used a filled circle as a cue in the inhibition conditions to indicate whether the first or second stimulus in each trial was relevant. This example illustrates trials with two-gap stimuli. A positive probe is presented in the control conditions; an intrusion probe is depicted in the access condition; and a positive probe is presented in the deletion condition. The text provides more details

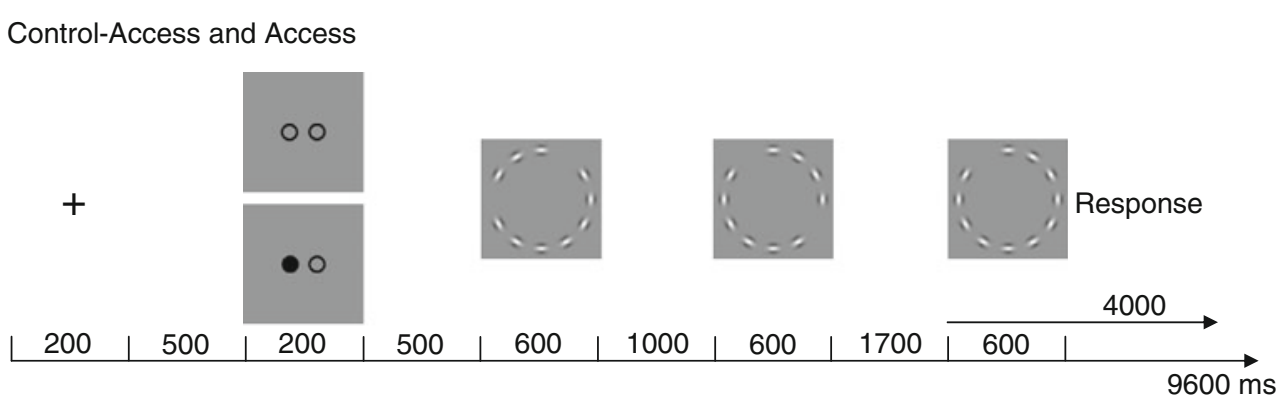

Control-Deletion and Deletion

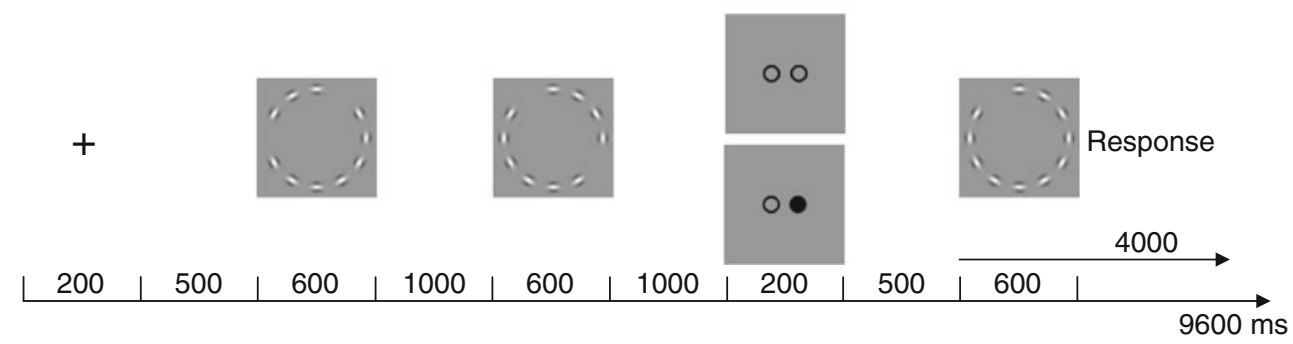

had errors to negative probes subtracted from them. The control conditions replicated the access and deletion conditions, except that in this case, the cues consisted of two empty circles, indicating that both stimuli were task relevant. The control conditions required storage, maintenance, and matching processes between the information in working memory and the probe. In the access and deletion conditions, subjects had to use these processes as well as interference control functions. Therefore, we independently evaluated access operations in selective attention and deletion functions in working memory rather than in conjunction with processes such as manipulation, updating, or transformation.

Furthermore, the task required that subjects see all of the events of a given trial to correctly identify the probe and provide their response. This aspect was especially important in the access condition, because there was an empty display between the test stimuli and the probe. Thus, confounding the probe without identifying all of the events in the trial was easy. Therefore, the access function in the current task consisted of filtering out or preventing the encoding of the irrelevant stimulus rather than avoiding seeing it. The evidence that subjects actually applied the access function should be reflected in better performance in the access condition than in its control condition, because only one stimulus was relevant for the task in the access condition, whereas both stimuli were relevant in the control condition. In addition, if subjects efficiently deactivated the memory trace of the irrelevant information, their memory should improve during the deletion condition relative to its respective control condition. Conversely, the finding that subjects did not improve their memory accuracy would suggest that memory traces for relevant and irrelevant information persisted and that subjects probably used an alternative strategy, such as remembering the gap positions of both stimuli but deciding based on only the relevant stimulus.

Finally, we sought to determine how the access and deletion functions affected working memory in relation to task difficulty by increasing the number of missing Gabors in the test stimuli. To investigate the inherent interaction of interference control and memory resources in each age group, it would be essential to manipulate task complexity (Conway \& Engle, 1994; Hasher \& Zacks, 1988).

\section{Method}

Subjects

Each age group consisted of 25 healthy subjects. The characteristics of the young-adult group (13 females, 12 males) and the older-adult group (9 females, 16 males) are listed in Table 1. All subjects were not addicted to alcohol or drugs and were free from neurological and psychiatric disorders. Young subjects received credits for their courses, and older adults received a financial payment as compensation for their participation in the study. All subjects provided informed consent before participation. The experiment was approved by the Bioethics Committee of the School of Psychology at the University of Padua. For inclusion in the study, subjects had to be either 21-30 years old (young subjects) or 61-75 years old (older subjects); have more than 12 years of education; have visual acuity that was normal or corrected to normal (20/20 with the Snellen chart); and have a Mini Mental State Exam (MMSE) score $\geq 28$ (Folstein, Folstein, \& McHugh, 1975), a Vocabulary scale (WAIS-R) score $\geq 8$ (Wechsler, 1981), and a Beck Depression Inventory (Beck, 1987) score 
Table 1 Subjects' characteristics and performance on neuropsychological tests

\begin{tabular}{|c|c|c|c|c|}
\hline & \multicolumn{2}{|c|}{ Young } & \multicolumn{2}{|l|}{ Old } \\
\hline & $M$ & $S D$ & $M$ & $S D$ \\
\hline Age (years) & 23.1 & 2.9 & 66.7 & 4.2 \\
\hline Education (years) & 16.3 & 1.6 & 16.3 & 3.0 \\
\hline Beck Depression Inventory & 6.0 & 5.6 & 6.4 & 4.4 \\
\hline Mini-Mental State & 29.6 & 0.7 & 29.3 & 1.0 \\
\hline Vocabulary Scale (WAIS-R) & 11.9 & 1.7 & 12.1 & 1.8 \\
\hline
\end{tabular}

$\leq 20$. The groups did not significantly differ $(p>.05)$ in duration of education or scores on the MMSE, the WAIS-R Vocabulary subtest, and the Beck Depression Inventory.

\section{Stimuli}

In each of the four experimental conditions, the stimuli consisted of circles created by circular cosine-phase Gabor elements - that is, the product of a sinusoidal grating and a Gaussian envelope (Fig. 1). The Gabors in the circle were oriented perpendicularly to its radius and were positioned in one of 12 possible locations corresponding to the numbers on a clock. The spatial frequency of the Gabor carrier was set to 3.2 cycles/degree, corresponding to a wavelength $(\lambda)$ of $0.31^{\circ}$, and then multiplied by a Gaussian envelope with a standard deviation $(\sigma)$ of $0.19^{\circ}$. The Gabors subtended a diameter visual angle of $0.41^{\circ}$, and the center-to-center distance between two contiguous Gabors in the circle was $0.82^{\circ}$. The circle of Gabors subtended a diameter visual angle of $3.60^{\circ}$. Three kinds of stimuli were created: circles with one, two, or three missing Gabors. In each case, the missing Gabors were not contiguous in the circle. Thus, a total of 12 stimuli were created in which one Gabor was missing (one-gap stimuli) in one of the 12 possible positions, 54 stimuli in which two Gabors were missing (two-gap stimuli), and 112 stimuli in which three Gabors were missing (three-gap stimuli). The stimuli with one gap were used as test and probe stimuli. The color of the screen background was gray during all the experiments, and its luminance $\left(50 \mathrm{~cd} / \mathrm{m}^{2}\right)$ was equal to the mean luminance of the Gabor element, which had a contrast of 0.77 cycles/ degree. The Gabors were created with MATLAB version 7.3.0.267 (R2006b) using the Psychophysics Toolbox extensions, version 3.0.8 beta (Brainard, 1997; Pelli, 1997).

In addition to the three Gabor stimuli, three types of cues were used: One was employed in the two control conditions (control-access and control-deletion), and two were used in the access and deletion conditions (Fig. 1). All cues consisted of two adjacent small circles presented simultaneously. In the control conditions, the circles were empty and conveyed no information to the subjects. This neutral cue was used only to create a sequence parallel to the sequence of events in the access and deletion conditions. In these conditions, two different cues were used: a blackfilled circle followed by an empty circle, or an empty circle followed by a black-filled circle. In both cases, the filled circle indicated whether the first or the second stimulus in each trial was relevant. The two contiguous circles in the cue subtended horizontal and vertical visual angles of $1.41^{\circ}$ and $0.57^{\circ}$, respectively. The diameter visual angle of each circle was $0.57^{\circ}$, with a contour line of $0.08^{\circ}$. The distance between the circles was $0.26^{\circ}$.

\section{Procedure}

Subjects participated in two sessions. In the first session, which was approximately an hour long, subjects performed the neuropsychological tests, and their visual acuity was measured using the Snellen chart. In the second session, subjects participated in four experimental conditions: control-access, access, control-deletion, and deletion. The second session took place in a dimly lit chamber and lasted about $3 \mathrm{~h}$ (including the resting intervals between conditions that were provided to all subjects). The controlaccess and access conditions were carried out in counterbalanced order, but contiguously; the same procedure was used for the control-deletion and deletion conditions. In addition, half of the subjects within each group participated first in the control-access and access conditions, followed by the control-deletion and deletion conditions, whereas the other half started in the inverse order. Before starting each condition, subjects participated in a practice session consisting of abbreviated versions of the experimental tasks. Subjects were seated at a distance of $70 \mathrm{~cm}$ from the monitor screen. At the end of this session, the contrast sensitivity function (obtained by plotting the reciprocal of binocular contrast threshold as a function of spatial frequency) was measured using the method of limits. This examination was performed at the same distance from the screen and with the same optical correction used for stimulus presentation during the experimental tasks and lasted about $5 \mathrm{~min}$. Note that at spatial frequencies of 2 cycles/degree (mean contrast threshold $\pm S D$ : young adults, $0.0019 \pm 0.0004$; older adults, $0.0030 \pm 0.002$ ), contrast thresholds were lower than the contrast $(0.77)$ used for the Gabor elements. The contrast sensitivity for both groups was within the norm for their age (Fig. 2). The experiment was controlled and behavioral responses were collected using the E-Prime software, version 1.2, from Psychology Software Tools, Inc.

From the complete set of stimuli assigned to each trial, stimuli were randomly selected for the four experimental conditions. For the two control conditions, 20 trials with 


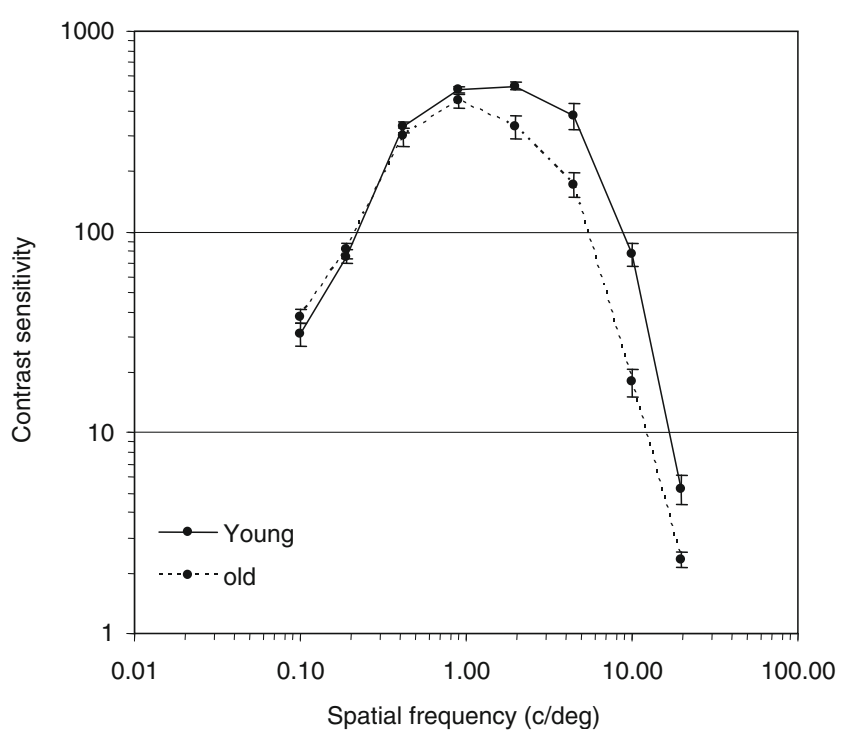

Fig. 2 Mean contrast sensitivity function for each age group

each type of stimulus (one, two, and three gaps) were generated. In addition, for each type of stimulus, 10 trials were created with positive probes (the position of the gap in the probe matched one of the positions of either of the two test stimuli) and 10 with negative probes (the position of the gap did not match the gaps presented in any of the two test stimuli). Positive probes matched the positions of each test stimulus in the same proportion. The total set of 60 trials was presented in a single block in random order. Two trials were added at the start of the block as fillers; the data from these trials were not analyzed.

In the access and deletion conditions, 240 trials were used: 80 trials with each type of stimulus (one, two, and three gaps). Three types of trials were generated for each kind of stimulus using positive, intrusion, or negative probes. Positive and negative probes were generated as previously described. Intrusion probes had a gap in the probe in the same position as a gap displayed in the irrelevant test stimulus. For each type of stimulus, 40 trials with positive probes, 20 trials with negative probes, and 20 trials with intrusion probes were generated. In half of each of these kinds of trials, the cue signaled that the first test stimulus was relevant, and, in the rest, the cue signaled that the second test stimulus was relevant. The total set of 240 trials was distributed in four blocks that included the same proportions of each type of trial. In addition, two filler trials were added at the beginning of each block; the data from these trials were not analyzed. The four blocks were presented in a different order to each subject.

Working memory paradigm by condition

Control-access Each trial began with the presentation of a small cross (horizontal and vertical visual angles of $0.61^{\circ}$ ) at the center of the screen for $200 \mathrm{~ms}$, indicating the visual focal point (Fig. 1). After a period of $500 \mathrm{~ms}$ in which the screen remained empty, a cue with two empty circles was presented for $200 \mathrm{~ms}$, followed by a blank screen that lasted $500 \mathrm{~ms}$. At the end of this period, the first and second test stimuli were displayed successively for $600 \mathrm{~ms}$, each separated by a $1,000-\mathrm{ms}$ interval during which the screen remained empty. A blank screen was displayed for $1,700 \mathrm{~ms}$ after the offset of the second test stimulus and was followed by the presentation of the probe, which was displayed for $600 \mathrm{~ms}$. Subjects were able to provide their response for a period of $4,000 \mathrm{~ms}$ after the onset of the probe. The screen remained empty after the presentation of the probe and until the beginning of the next trial. The interval between successive trials was $9.6 \mathrm{~s}$. The stimulus exposition time and the intertrial interval were determined after several pilot studies, which demonstrated that these periods provided subjects enough time to visualize each stimulus and prepare for the next trial after providing their response. Subjects were instructed to judge whether the gap position of the probe matched the position of one of the gaps displayed in either of the two test stimuli. Subjects responded by pressing one of two possible keys on a computer keyboard; the keys (" $\mathrm{z}$ " and " $\mathrm{m}$ ") were pressed by the subjects' left and right index fingers, and the assignment key for the two possible responses (same or different gap) was counterbalanced across subjects.

Access The paradigm was identical to the one used in the control-access condition. However, the filled circle in the cue indicated to the subject which of the two test stimuli was relevant for the specific trial. Subjects were requested to judge whether the gap located in the probe matched any of the gaps presented in the previous relevant test stimulus for the specific trial.

Control-deletion The events and the duration of each of these trials were equal to those from the control-access condition, except that the cue was presented 1,000 ms after the offset of the second test stimulus (Fig. 1). The subjects' task was identical to the one used in the control-access condition.

Deletion Trials were presented as in the control-deletion condition, but, as in the access condition, the filled circle in the cue indicated to the subject which of the two test stimuli was relevant in each trial.

Data analysis

Mixed ANOVAs were performed, followed by post hoc cell mean tests when interactions turned out to be significant, as 
proposed by Toothaker (1993). To compensate for inhomogeneous covariances that might produce marked increases in Type I errors, the degrees of freedom were corrected using the Greenhouse-Geisser procedure. For these cases, the original degrees of freedom, the Greenhouse-Geisser coefficient $(\varepsilon)$, and the corrected probability levels are reported. In addition, the effect size was calculated for all analyses using the partial $\eta^{2}\left(\eta_{\mathrm{p}}^{2}\right)$ statistic. The significance level was $p<.05$.

\section{Results}

\section{Inhibition effects}

Inhibition effects were examined by measuring the performance difference between each experimental condition and its respective control condition. The mixed ANOVAs for both accuracy and reaction times in correct responses included the factors Group, Inhibition Condition (access = difference between access and control-access conditions; deletion = difference between deletion and control-deletion conditions), and Number of Gaps. The analysis for accuracy was significant for the factors Group $\left[F(1,48)=15.64, p<.001, \eta_{\mathrm{p}}^{2}=.25\right]$, Inhibition Condition $\left[F(1,48)=20.23, p<.001, \eta_{\mathrm{p}}^{2}=.30\right]$, and Number of Gaps $[F(2,96)=32.92, p<.001, \varepsilon=.77$, $\left.\eta_{\mathrm{p}}^{2}=.41\right]$, as well as for the interactions between the factors Group and Inhibition Condition $[F(1,48)=10.66, p=.002$, $\left.\eta_{\mathrm{p}}^{2}=.18\right]$ and Inhibition Condition and Number of Gaps $[F$ $\left.(2,96)=4.46, p=.02, \varepsilon=.93, \eta_{\mathrm{p}}^{2}=.9\right]$ (Fig. 3). Post hoc mean cell tests revealed that deletion effects differed between groups (young adults [mean $\pm S E$ ], $9.2 \pm 1.4$; older adults, $0.2 \pm 1.4$ ) but not access effects (young adults, $10.9 \pm 1.1$; older adults, $10.8 \pm 1.1$ ). In addition, access and deletion effects differed in older adults but not in young adults. Access effects were higher than deletion effects in trials with two-gap (access, 18.2 \pm 1.5 ; deletion, 9.1 \pm 1.8 ) and three-gap (access, $11.8 \pm 1.5$; deletion, $4.3 \pm 1.7$ ) stimuli, but not in trials with one-gap stimuli (access, $2.6 \pm 0.9$; deletion, $0.7 \pm 1.1$ ).

An additional analysis was conducted to test whether these inhibition effects differed from zero. This analysis $\left[F(1,48)=10.66, p=.002, \eta_{\mathrm{p}}^{2}=.18\right]$ revealed that the access effects differed significantly from zero in both age groups, but the deletion effects differed from zero only in young adults, according to post hoc mean cell tests. Besides $\left[F(2,96)=4.46, p=.02, \varepsilon=.93, \eta_{\mathrm{p}}^{2}=.09\right]$, access effects differed from zero in trials with two-gap and three-gap stimuli, but deletion effects only in trials with two-gap stimuli, according to the post hoc mean cell test.

The effects of inhibition on reaction times were examined by measuring the difference between each inhibition condition and its respective control condition. Reaction times during correct responses were log transformed to test age differences under proportional measurements (Oberauer, 2001). The factors Inhibition Condition $\left[F(1,48)=18.71, p<.001, \eta_{\mathrm{p}}^{2}=.28\right]$ and Number of Gaps $[F(2,96)=23.09, p<.001, \varepsilon=.95$, $\left.\eta_{\mathrm{p}}^{2}=.33\right]$ and the interaction between these two factors were significant $\left[F(2,96)=6.23, p=.004, \varepsilon=.93, \eta_{\mathrm{p}}^{2}=.12\right]$ (Fig. 4). The factor Group $[F(1,48)=2.39, p=.13$, $\left.\eta_{\mathrm{p}}^{2}=.05\right]$ and the interactions Group $\times$ Inhibition Condition $\left[F(1,48)=0.25, p=.62, \eta_{\mathrm{p}}^{2}=.01\right]$, Group $\times$ Number of Gaps $\left[F(2,96)=2.32, p<.11, \varepsilon=.95, \eta_{\mathrm{p}}^{2}=.05\right]$, and Group $\times$ Inhibition Condition $\times$ Number of Gaps $[F(2$, $\left.96)=0.83, p<.43, \varepsilon=.93, \eta_{\mathrm{p}}^{2}=.02\right]$ were not significant. Post hoc mean cell tests indicated that inhibition conditions differed significantly in trials with one-gap (access, $-157 \pm 22$; deletion, $45 \pm 29$ ) and two-gap (access, $-221 \pm 32$; deletion, $-59 \pm 34$ ) stimuli, but not in trials with three-gap stimuli (access, $-36 \pm 33$; deletion, $37 \pm 35$ ). This same analysis conducted on raw reaction time data yielded results identical to those obtained in the log-transformed data.

The analyses conducted to test whether these inhibition effects on reaction times differed from zero were significant $\left[F(2,96)=6.23, p=.004, \varepsilon=.93, \eta_{\mathrm{p}}^{2}=.12\right]$. The post hoc mean cell test indicated that only the access effects in trials with one-gap and two-gap stimuli differed significantly from zero.

\section{Intrusion effects}

Intrusion effects were examined in terms of the difference between incorrect responses to intrusion probes and negative probes. The analysis included the factors Group, Inhibition Condition (access and deletion), and Number of Gaps. The factors Group $\left[F(1,48)=10.62, p=.002, \eta_{\mathrm{p}}^{2}=.18\right]$, Inhibition Condition $\left[F(1,48)=25.46, p<.001, \eta_{\mathrm{p}}^{2}=.35\right]$, and Number of Gaps $[F(2,96)=75.18, p<.001, \varepsilon=.96$, $\left.\eta_{\mathrm{p}}^{2}=.61\right]$, as well as the interaction between the factors Group and Inhibition Condition $[F(1,48)=10.64, p=.002$, $\left.\eta_{\mathrm{p}}^{2}=.18\right]$, were significant (Fig. 5). Post hoc mean cell tests revealed that intrusion effects differed significantly between groups in the deletion condition (young adult, $3.7 \pm 1.5$; older adults, $12.6 \pm 1.5$ ) but not in the access condition (young adult, $1.3 \pm 1.2$; older adults, $1.7 \pm 1.2$ ). In addition, intrusion effects differed between conditions in older adults but not in young adults. Intrusion effects were equivalent in trials with one-gap $(3.2 \pm 0.8)$ and two-gap $(-4.0 \pm 1.4)$ stimuli, but they differed between these two kinds of trials and trials with three-gap stimuli $(15.3 \pm 1.2)$. Intrusion effects on reaction times were not examined, because not all subjects produced incorrect responses in all kinds of trials.

\section{Regression analyses}

Regression analyses were conducted to test whether the visuospatial task induced proactive interference or fatigue. 
Fig. 3 Mean percent correct responses in young and older adults as a function of task difficulty in the access and deletion conditions and their respective control conditions. Error bars represent standard errors

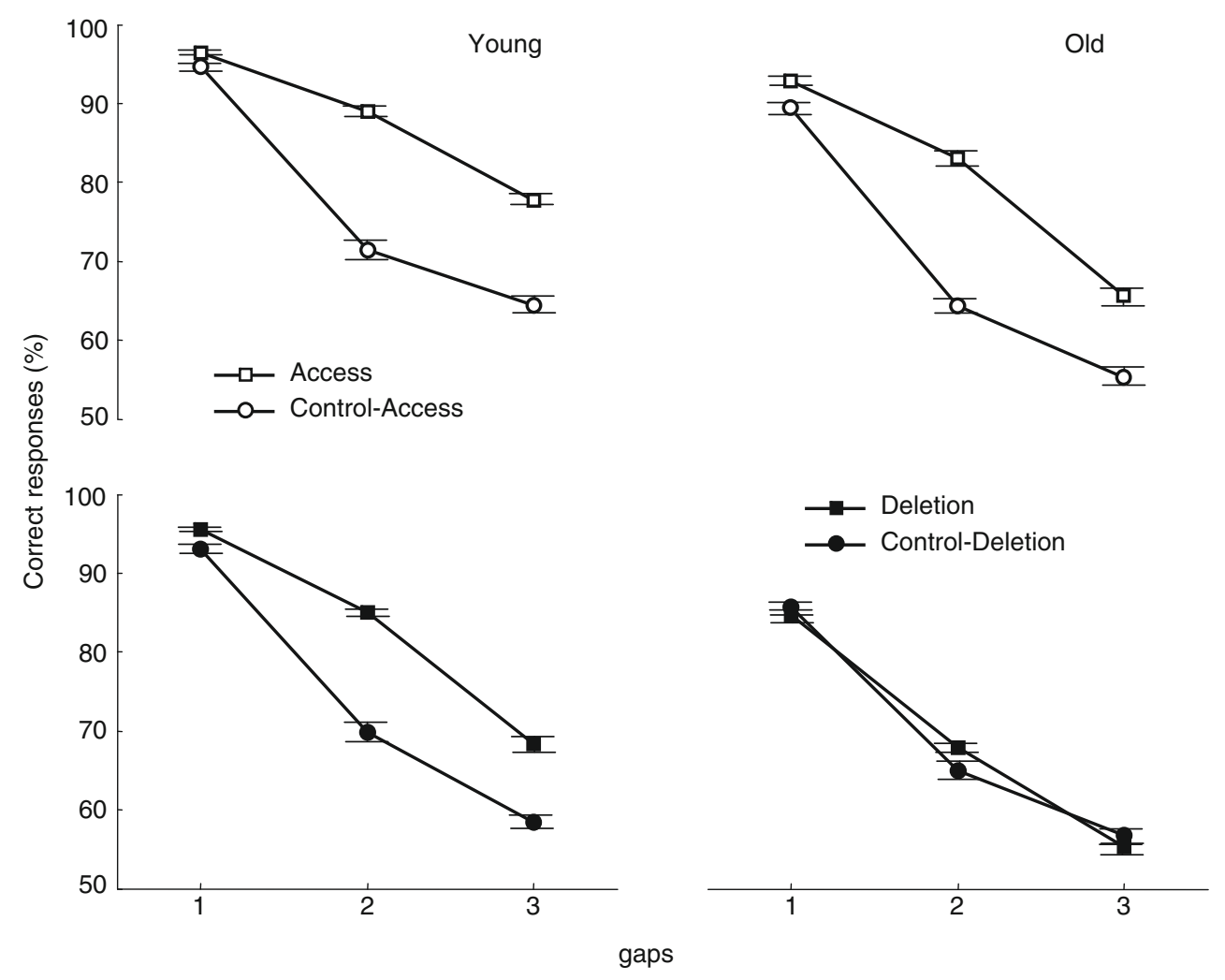

The accuracy trial by trial was examined across each of the 60 trials from the control conditions and each of the 240 trials from the access and deletion conditions in order to assess whether the performance of the subjects diminished across trials in the order they were administered. The performance in each trial was averaged separately for each age group. The proportion of the variance in accuracy predicted by trial order was not significant in the inhibition conditions (access: young
Fig. 4 Mean reaction times in young and older adults as a function of task difficulty in the access and deletion conditions and their respective control conditions. Error bars represent standard errors

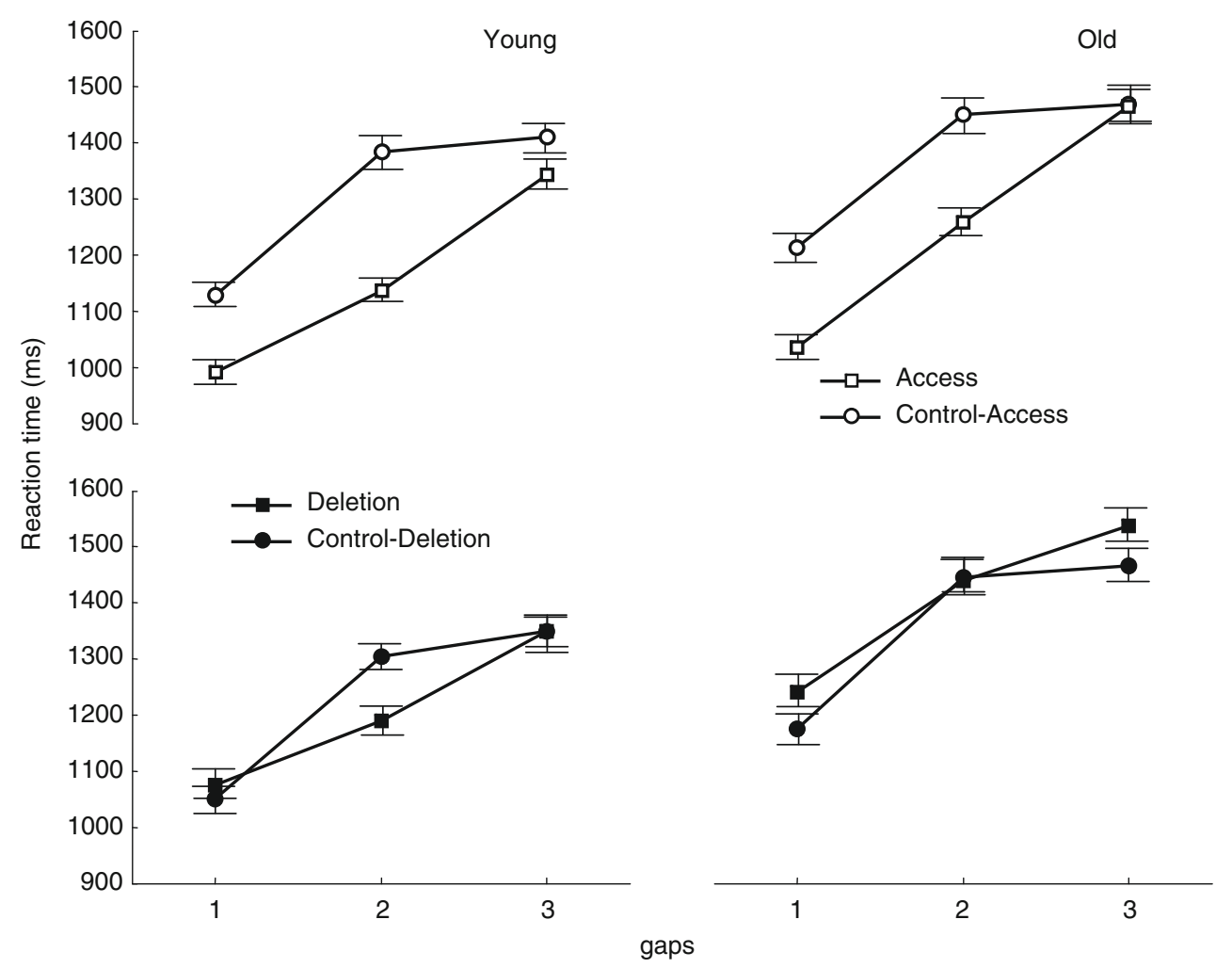


Fig. 5 Mean percent intrusion effects (difference between errors to intrusion probes and to negative probes) in young and older adults as a function of task difficulty in the access and deletion conditions. Error bars represent standard errors

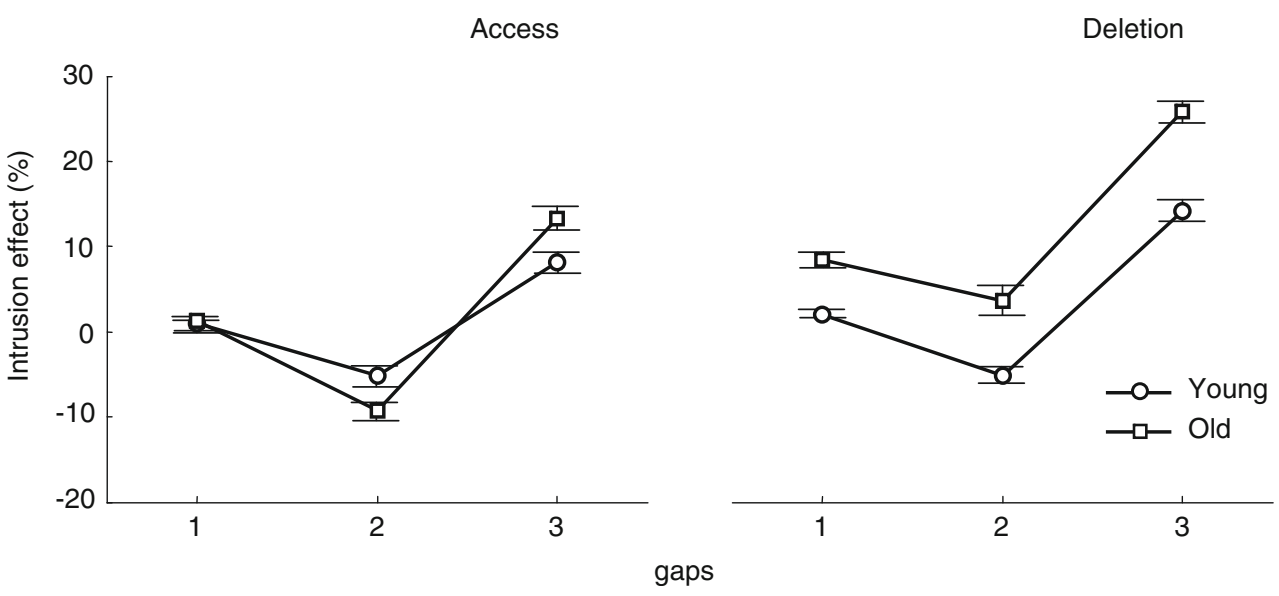

adults, $R^{2}=.0002, p=.81$; older adults, $R^{2}=.006, p=.24$; deletion: young adults, $R^{2}=.002, p=.49$; older adults, $R^{2}=$ $.0005, p=.74$ ), or in the control conditions (control-access: young adults, $R^{2}=.03, p=.21$; older adults, $R^{2}=.002, p=$ .77; control-deletion: young adults, $R^{2}=.003, p=.70$; older adults, $R^{2}=.02, p=.26$ ).

\section{Discussion}

The present results demonstrate that older adults were as efficient as young adults in applying the access functions but were unable to make use of the deletion functions. Both age groups improved their memory performance by approximately $11 \%$ when they were requested to use the access functions, relative to their performance in the control-access condition, in which no interference regulation was required. Additionally, intrusion effects in the access condition were less than $2 \%$ for both age groups. Conversely, the performance of older adults in the deletion condition matched their performance in the control-deletion condition, indicating that older adults, unlike younger adults, did not use internal interference control mechanisms to improve their memory. Younger adults improved their memory by approximately $9 \%$ relative to their performance in the control-deletion condition. The large size of the intrusion effects (approximately nine percentage points higher than for younger adults) provides further evidence that older adults have poor control of their deletion functions.

The only previous researchers (Dumas \& Hartman, 2008) who had contrasted both mechanisms of inhibition under equivalent circumstances in young and older adults found no evidence of age difference in either the access or deletion functions of inhibition. Thus, the present study confirms the finding that the ability to control external distractors does not change with age. This conclusion has also gained strong support when these mechanisms have been evaluated by the Stroop task, as revealed by a meta- analysis of 20 studies (Verhaeghen \& De Meersman, 1998), which demonstrated that the interference effect observed in this task is merely the result of the classical slowing of cognitive processing with advancing age (Salthouse, 1996). The domains used to evaluate these functions might explain the lack of concord observed for the deletion function results between our study and Dumas and Hartman. The latter study used verbal information, which can be influenced by variables such as reading skills (Chiappe et al., 2000; De Beni et al., 1998) or the semantic relationships between stimuli (which produce more interference than words that are semantically unrelated; Connelly et al., 1991). In fact, Dumas and Hartman also evaluated interference effects using semantically distracting words related to target words to increase the level of interference. They found that the working memory performance of older adults was better when words were semantically related than when they were unrelated. This result shows that the use of words as stimuli to evaluate interference effects involves variables that are difficult to control.

Several cognitive control mechanisms may be responsible for the improvement in memory accuracy in the access condition relative to its control condition. One possibility is that inhibition mechanisms control the interference from irrelevant stimuli (i.e., subjects actively suppress or avoid encoding irrelevant stimuli into their working memory; Hasher et al., 1999; Wilson \& Kipp, 1998). Alternatively, subjects might take advantage of the prestimulus cue to selectively attend to relevant stimuli without acting on irrelevant stimuli. Accordingly, discriminating between relevant and irrelevant stimuli, selecting relevant information, enhancing memory representations, and maintaining the active representation in working memory until the goal had been achieved controlled interference (Kane et al., 2007; Unsworth \& Engle, 2007). In fact, the presentation of a cue before or after the stimulus is insufficient to discern whether subjects applied an inhibition mechanism or merely ignored the irrelevant stimulus and focused only on the relevant information. The efficient differentiation 
between relevant and irrelevant stimuli is the only process necessary either to inhibit or select information.

An inhibition account of the successful control of the access functions is difficult to support in an early processing stage such as selective attention, because the task requires the efficient selection and maintenance of relevant information, whereas the active suppression of irrelevant information is not essential and may only be a waste of resources. In addition, interference control in selective attention is related to enhancing relevant information rather than inhibiting irrelevant information (MacLeod, Dodd, Sheard, Wilson, \& Bibi, 2003). Alternatively, the irrelevant stimulus might have elicited such low interference effects in both age groups that no direct action had to be taken to inhibit this information. Conversely, when interference is high (as demonstrated by lower memory accuracy/longer reaction times in older adults relative to young adults), inhibition mechanisms may be necessary. Two event-related potential studies have shown that older adults are more susceptible to distracting information presented either separately from (Gazzaley, Clapp, McEvoy, Knight, \& D'Esposito, 2008) or along with (De Fockert, Ramchurn, Van Velzen, Bergström, \& Bunce, 2009) relevant information, as compared to younger adults. This finding suggests that the decline in memory performance observed in older adults is due to a greater amount of irrelevant information processing, and thus, to a failure in inhibition.

The present results show an inability of older adults to apply deletion functions in the visuo-spatial domain, a result that contradicts the lack of age differences observed in the only previous study that evaluated these mechanisms in the spatial domain (Cornoldi et al., 2007). However, the different results between our study and the previous one might be explained by the fact that the task employed by Cornoldi et al., similar to the reading span task, might have involved a combination of access and deletion functions, because in both tasks subjects are instructed in advance to remember only the last position of each matrix or only the last word of each sentence, respectively. This instruction clearly activates the access functions in addition to the deletion functions, because in these tasks subjects are compelled to see all positions signaled in the matrices or to read all words in each sentence, respectively.

The impairment of several cognitive control processes might underlie older adults' inability to improve their memory accuracy in the deletion condition relative to its control condition. One possibility is that older adults are incapable of inhibiting the internal representation of irrelevant stimuli and preserving only useful information in working memory. Thus, the presence of relevant and irrelevant memories increased competition and confusion. However, the poststimulus cue might provide little benefit to older adults because they are unable to selectively attend to memories of relevant stimuli. Moreover, older adults might have been unable to distinguish between relevant and irrelevant memories in the first place, affecting the inhibition as well as the selection of memories. Indeed, the inhibition of distracting memories and the selection of relevant memories are related to internal interference control (Anderson \& Neely, 1996; Bjork, 1989). Thus, our results suggest that memory accuracy in older adults did not benefit from the poststimulus cue because they were unable to apply one or both of these cognitive control processes.

Alternatively, older adults might have been unable to choose an appropriate strategy to solve the task. After the experimental session, we asked subjects about the strategies they used to solve the task. They communicated that using a numerical code to remember the positions of the missing Gabors (e.g., coding the positions as in numbers on a clock) was impossible, because there was not enough time. Instead, most subjects reported that they used a visual strategy - for example, mentally tracing lines or triangles between the gaps without considering their exact positions, but only their relative positions. Although both age groups used this strategy, older adults might have received less benefit from it, because older adults perform worse than younger adults on visual search tasks (Madden, Spaniol, Bucur, \& Whiting, 2007; Madden et al., 2002) and visual memory tasks (Bopp \& Verhaeghen, 2007).

Moreover, the failure of the deletion functions observed in older adults was evident at all complexity levels, because the age effect on inhibition and intrusion did not interact with task difficulty. This finding suggests that the deletion functions were impaired even when the memory task demanded minimal resources (Conway \& Engle, 1994) or, as was proposed in the framework of Hasher and Zacks (1988), when competition between relevant and irrelevant information was least. As in the present study, De Beni and Palladino (2004) found that deletion functions were less efficient in older adults relative to younger adults across two levels of difficulty. Thus, the inability of elderly subjects to apply deletion functions seems to be a qualitative difficulty rather than a quantitative deficit. Older adults were less capable of actively suppressing memory traces for irrelevant information and actively selecting memory traces for relevant information. We observed this outcome in spite of the fact that task difficulty significantly increased intrusion effects in high-difficulty (three-gap) trials relative to low- (one-gap) and moderate- (two-gap) difficulty trials for both access and deletion mechanisms. These results confirm that both interference control functions are vulnerable when the amount of information that is required to be stored to accomplish the memory task increases; however, this vulnerability does not increase with age. 
The memory task employed in the present study allowed us to examine both access and deletion functions free from the influence of proactive interference. Performance in the reading span task, for example, has the disadvantage of being sensitive to proactive interference, which obscures its interpretation, which was empirically confirmed when the classical procedure of increasing the number of sentences in each set to examine working memory capacity was inverted by presenting first the sets with the largest number of sentences and progressing to smaller sets (Lustig et al., 2001; May et al., 1999). The present study examined trialby-trial accuracy via a regression analysis to provide evidence that proactive interference did not accumulate in any experimental conditions. Proactive interference was not expected to build up in the visuospatial paradigm used here, because the circles of Gabor stimuli were practically identical (with the exception of the numbers of gaps and their position), making them difficult to remember across trials. The use of meaningless stimuli also limited possible associations based on prior knowledge and prevented the prolongation of the memory trace after the trial in which it was useful.

The effects of either access or deletion functions on reaction times (i.e., the differences between inhibition and control conditions) were similar between age groups. This result indicates that the visuospatial task employed in the present study was insensitive to detecting the age-related processing-speed decline that has been extensively confirmed (Salthouse, 1991). Beneficial effects on reaction times were evident in both groups only when subjects applied the access function relative to its control condition, specifically in trials with one- and two-gap stimuli, because access effects on reaction times in these trials differed significantly from zero. The results suggest that when memory load is equal to or greater than three gaps, the process of matching the gap positions retained in memory with the gap presented in the probe becomes so demanding that no improvement in processing speed is possible. This was evident because reaction times were approximately the same when subjects from both age groups successfully applied the access functions in three-gap trials (thus, reducing the memory load to only three gap positions; $1,405 \mathrm{~ms}$ ) and when the memory load increased to four (two-gap trials; $1,418 \mathrm{~ms}$ ) and six (three-gap trials; $1,441 \mathrm{~ms})$ gap positions in the control-access condition. Alternatively, subjects may have experienced uncertainty when they were required to retain three or more gap positions, which could have prevented any benefits to reaction times, when they applied the access functions in three-gap trials. These difficulties were also encountered in trials with three-gap stimuli in the deletion condition, because the access and deletion effects on the reaction times differed only on trials with one- and two-gap stimuli.
The time between the onset of the cue and the probe $(700 \mathrm{~ms})$ in the deletion condition was sufficient for subjects to be able to apply the deletion functions. Oberauer (2001, Exp. 1) tested the amount of time necessary to remove irrelevant information from the focus of attention by varying the time between cue and probe. The set size of irrelevant information did not affect the reaction times of younger and older adults after $600 \mathrm{~ms}$, indicating that both groups removed irrelevant information from their working memory after this period of time. This finding suggests that the delay between the cue and the probe in the present study was sufficient for both age groups to activate the deletion functions. In addition, the fact that older and younger adults provided responses in approximately equal amounts of time shows that the older adults' inability to apply the deletion functions was not due to the lack of time or an increase in uncertainty. Because young adults apply deletion functions (as their improvements in accuracy in the deletion condition relative to its control condition reveal), we also expected improved reaction times after their memory load was reduced. However, younger adults' reaction times did not decrease. This outcome suggests that deletion functions are time consuming, and for this reason they are able to benefit memory accuracy but not the speed of processing. Therefore, the time required by subjects to control memory-based interference and provide their responses in the deletion condition was equivalent to the time subjects required to retain both stimuli in memory and to decide their responses in the control-deletion condition.

Access effects benefited working memory performance more than deletion effects in trials with two- and three-gap stimuli in both age groups. Other studies observed the same finding when contrasting interference control functions in younger (Dumas \& Hartman, 2008; Nee \& Jonides, 2008, 2009) and older subjects (Dumas \& Hartman, 2008). One possible explanation for this result may be that access functions operate during selective attention; therefore, these mechanisms do not require resources from working memory, which leads to a higher memory benefit. Conversely, because the deletion functions occur after information enters working memory, these mechanisms share resources with the other processes that occur simultaneously in working memory. However, selective attention and working memory may not be independent processes. In fact, there is evidence that increasing memory load in a working memory task augments the time younger subjects spend processing distractor stimuli in a selective attention task (De Fockert, Rees, Frith, \& Lavie, 2001). In the present study, the intrusion effects for both inhibition conditions were significantly higher in trials with higher memory loads (three-gap trials) as compared to less demanding memory loads (one- and two-gap trials). This result could mean that access functions also depend on 
working memory resources; however, a more plausible explanation is that some irrelevant information enters working memory when subjects apply these functions, and this information, regardless of task difficulty, causes more interference when memory load is high because representations become fragile and more vulnerable to interference.

The comparison of access and deletion functions with their respective control conditions allowed us to directly examine the effects of these functions on working memory performance. Previous studies (Andrés et al., 2004; Dumas \& Hartman, 2008) included a control condition, but only one of them (Andrés et al., 2004) found deletion effect differences between younger and older adults. In the present study, we found evidence that younger adults applied both interference control functions, whereas older adults applied only the access functions, because subjects improved their memory performance when they used these mechanisms relative to their respective control conditions. The possibility that subjects applied the same strategy in access and deletion conditions that they applied in the respective control conditions is unlikely, given the present results. The strategy of remembering both test stimuli without inhibiting or selecting them and remembering the cue information regarding the relevant stimuli (i.e., "remember everything and discriminate later" strategy) would have increased the difficulty in the inhibition conditions relative to the respective control conditions. This is because under access and deletion conditions, subjects were required to discriminate between relevant and irrelevant stimuli, a process that was not required in the control conditions.

Neuroimaging studies (Nee \& Jonides, 2008, 2009) in young adults provide evidence that both processes, the selection of percepts and the selection of memories in the presence of competing distractors, depend on several common brain regions, such as the right dorsolateral prefrontal cortex and certain parietal regions. However, some brain regions are activated uniquely during each of these processes. The occipital cortex is activated when subjects control interference by filtering percepts, whereas the left lateral prefrontal cortex is activated when subjects control interference by filtering intrusive memories (Nee \& Jonides, 2008). In the present study, that older subjects applied the access functions efficiently but were incapable of employing the deletion functions suggests that different cognitive control functions underlie these abilities. This finding provides support for the proposal that multiple independent mechanisms control interference (Braver et al., 2007; Friedman \& Miyake, 2004; Lustig, Hasher, \& Zacks, 2007) rather than one interference control function. However, in spite of the fact that these interference control functions rely on partially different mechanisms, they are not completely different or independent.
Acknowledgements We are grateful to M. Paradiso, C. Venturini, S. Cosentino, and S. Castelli for assistance in the experiments. S.C. was supported by CONACYT $(80376,98801)$ and by DGAPA, National Autonomous University of Mexico (PASPA, PRIDE IN300309).

\section{References}

Anderson, M. C., \& Bjork, R. A. (1994). Mechanisms of inhibition in long-term memory: A new taxonomy. In D. Dagenbach \& T. Carr (Eds.), Inhibitory processes in attention, memory and language (pp. 265-326). San Diego: Academic Press.

Anderson, M. C., \& Neely, J. H. (1996). Interference and inhibition in memory retrieval. In E. L. Bjork \& R. A. Bjork (Eds.), Memory (Handbook of perception and cognition) (2nd ed., pp. 237-313). San Diego: Academic Press.

Andrés, P., Van der Linden, M., \& Parmentier, F. B. R. (2004). Directed forgetting in working memory: age-related differences. Memory, 12, 248-256.

Beck, A. T. (1987). Beck depression inventory. San Antonio: Psychological Corp.

Bjork, R. A. (1989). Retrieval inhibition as an adaptive mechanism in human memory. In H. L. Roediger III \& F. I. M. Craik (Eds.), Varieties of memory and consciousness: essays in honor of Endel Tulving (pp. 309-330). Hillsdale: Erlbaum.

Bopp, K. L., \& Verhaeghen, P. (2007). Age-related differences in control processes in verbal and visuo-spatial working memory: Storage, transformation, supervision, and coordination. Journals of Gerontology, 62B, P239-P246.

Brainard, D. H. (1997). The psychophysics toolbox. Spatial Vision, 10, 433-436.

Braver, T. S., Gray, J. R., \& Burgess, G. C. (2007). Explaining the many varieties of working memory variation: Dual mechanisms of cognitive control. In A. R. A. Conway, C. Jarrold, M. J. Kane, A. Miyake, \& J. N. Towse (Eds.), Variation in working memory (pp. 76-106). New York: Oxford University Press.

Cansino, S. (2009). Episodic memory decay along the adult lifespan: a review of behavioral and neurophysiological evidence. International Journal of Psychophysiology, 71, 64-69.

Carlson, M. C., Hasher, L., Connelly, S. L., \& Zacks, R. T. (1995). Aging, distraction, and the benefits of predictable location. Psychology and Aging, 10, 427-436.

Chiappe, P., Hasher, L., \& Siegel, L. S. (2000). Working memory, inhibitory control, and reading disability. Memory \& Cognition, $28,8-17$.

Clark, J. M. (1996). Contributions of inhibitory mechanisms to unified theory in neuroscience and psychology. Brain and Cognition, 30, $127-152$

Cohn, N. B., Dustman, R. E., \& Bradford, D. C. (1984). Age-related decrements in stroop color test performance. Journal of Clinical Psychology, 40, 1244-1250. doi:10.1002/1097-4679(198409) 40:5<1244::AID-JCLP2270400521>3.0.CO;2-D.

Comalli, P. E., Wapner, S., \& Werner, H. (1962). Interference effects of Stroop color-word test in childhood, adulthood, and aging. The Journal of Genetic Psychology, 100, 47-53.

Connelly, S. L., Hasher, L., \& Zacks, R. T. (1991). Age and reading: the impact of distraction. Psychology and Aging, 6, $533-541$.

Conway, A. R. A., \& Engle, R. W. (1994). Working memory and retrieval: A resource-dependent inhibition model. Journal of Experimental Psychology. General, 123, 354-373.

Cornoldi, C., Bassani, C., Berto, R., \& Mammarella, N. (2007). Aging and the intrusion superiority effect in visuo-spatial working memory. Aging, Neuropsychology, and Cognition, 14, $1-21$. 
Daneman, M., \& Carpenter, P. A. (1980). Individual differences in working memory and reading. Journal of Verbal Learning and Verbal Behavior, 19, 450-466.

De Beni, R., \& Palladino, P. (2004). Decline in working memory updating through ageing: Intrusion error analyses. Memory, 12, 75-89.

De Beni, R., Palladino, P., Pazzaglia, P., \& Cornoldi, C. (1998). Increases in intrusion errors and working memory deficit of poor comprehenders. The Quarterly Journal of Experimental Psychology, 51A, 305-320.

De Fockert, J., Ramchurn, A., Van Velzen, J., Bergström, Z., \& Bunce, D. (2009). Behavioural and ERP evidence of increased interference in old age. Brain Research, 1282, 67-73.

De Fockert, J., Rees, G., Frith, C. D., \& Lavie, N. (2001). The role of working memory in visual selective attention. Science, 291, $1803-1806$

Dumas, A. J., \& Hartman, M. (2008). Adult age differences in the access and deletion functions of inhibition. Aging, Neuropsychology, and Cognition, 15, 330-357.

Folstein, M. F., Folstein, S. E., \& McHugh, P. R. (1975). Mini-mental state. Journal of Psychiatric Research, 12, 189-198.

Friedman, N. P., \& Miyake, A. (2004). The relations among inhibition and interference cognitive functions: a latent variable analysis. Journal of Experimental Psychology. General, 133, 101-135.

Gamboz, N., Russo, R., \& Fox, E. (2002). Age differences and the identity negative priming effect: an updated meta-analysis. Psychology and Aging, 17, 525-531.

Gazzaley, A., Clapp, W., McEvoy, K., Knight, R., \& D'Esposito, M. (2008). Age-related top-down suppression deficit in the early stages of cortical visual memory processing. Proceedings of the National Academy of Sciences, 105, 13122-13126.

Hartman, M., \& Hasher, L. (1991). Aging and suppression: memory for previously relevant information. Psychology and Aging, 6, $587-594$.

Hasher, L., Stoltzfus, E. R., Zacks, R. T., \& Rypma, B. (1991). Age and inhibition. Journal of Experimental Psychology. Learning, Memory, and Cognition, 17, 163-169.

Hasher, L., \& Zacks, R. T. (1988). Working memory, comprehension, and aging: A review and a new view. In G. H. Bower (Ed.), The psychology of learning and motivation: Advances in research and theory (pp. 193-225). San Diego: Academic Press.

Hasher, L., Zacks, R. T., \& May, C. P. (1999). Inhibitory control, circadian arousal, and age. In D. Gopher \& A. Koriat (Eds.), Attention and performance XVII: Cognitive regulation of performance. Interaction of theory and application (pp. 653-675). Cambridge: MIT Press.

Houx, P. J., Jolles, J., \& Vreeling, F. W. (1993). Stroop interference: aging effects assessed with the Stroop color-word test. Experimental Aging Research, 19, 209-224.

Kane, M. J., Conway, A. R. A., Hambrick, D. Z., \& Engle, R. W. (2007). Variation in working-memory capacity as variation in executive attention and control. In A. R. A. Conway, C. Jarrold, M. J. Kane, A. Miyake, \& J. Towse (Eds.), Variation in working memory (pp. 21-48). New York: Oxford University Press.

Kane, M. J., Hasher, L., Stoltzfus, E. R., Zacks, R. T., \& Connelly, S. L. (1994). Inhibitory attentional mechanisms and aging. Psychology and Aging, 9, 103-112.

Kieley, J. M., \& Hartley, A. A. (1997). Age-related equivalence of identity suppression in the Stroop color-word task. Psychology and Aging, 12, 22-29. doi:10.1037/0882-7974.12.1.22.

Kramer, A. F., Humphrey, D. G., Larish, J. F., \& Logan, G. D. (1994). Aging and inhibition: beyond a unitary view of inhibitory processing in attention. Psychology and Aging, 9, 491-512. doi:10.1037/0882-7974.9.4.491.

Lustig, C., Hasher, L., \& Zacks, R. T. (2007). Inhibitory deficit theory: Recent developments in a "new view. In D. S. Gorfein \& C. M.
MacLeod (Eds.), The place of inhibition in cognition (pp. 145162). Washington, DC: American Psychological Association.

Lustig, C., May, C. P., \& Hasher, L. (2001). Working memory span and the role of proactive interference. Journal of Experimental Psychology. General, 130, 199-207.

MacLeod, C. M. (2007). The concept of inhibition in cognition. In D. S. Gorfein \& C. M. MacLeod (Eds.), inhibition in cognition (pp. 3-23). Washington, DC: American Psychological Association.

MacLeod, C. M., Dodd, M. D., Sheard, E. D., Wilson, D. E., \& Bibi, U. (2003). In opposition to inhibition. In B. H. Ross (Ed.), The psychology of learning and motivation (pp. 163-214). San Diego: Academic Press.

Madden, D. J., Spaniol, J., Bucur, B., \& Whiting, W. L. (2007). Agerelated increase in top-down activation of visual features. The Quarterly Journal of Experimental Psychology, 60, 644-651. doi:10.1080/17470210601154347.

Madden, D. J., Turkington, T. G., Provenzale, J. M., Denny, L. L., Langley, L. K., Hawk, T. C., et al. (2002). Aging and attentional guidance during visual search: functional neuroanatomy by positron emission tomography. Psychology and Aging, 17, 24 43. doi:10.1037/0882-7974.17.1.24.

May, C. P., Hasher, L., \& Kane, M. J. (1999). The role of interference in memory span. Memory \& Cognition, 27, 759-767.

McDowd, J. M., \& Filion, D. L. (1995). Aging and negative priming in a location suppression task: the long and the short of it. Psychology and Aging, 10, 34-47. doi:10.1037/08827974.10.1.34.

Miller, E. K., \& Cohen, J. D. (2001). An integrative theory of prefrontal cortex function. Annual Review of Neuroscience, 24, 167-202.

Nee, D. E., \& Jonides, J. (2008). Dissociable interference-control processes in perception and memory. Psychological Science, 19, 490-500.

Nee, D. E., \& Jonides, J. (2009). Common and distinct neural correlates of perceptual and memorial selection. Neuroimage, 45, 963-975.

Neill, W. T., \& Valdes, L. A. (1992). Persistence of negative priming: steady state or decay? Journal of Experimental Psychology. Learning, Memory, and Cognition, 18, 565-576. doi:10.1037/ 0278-7393.18.3.565.

Nigg, J. T. (2000). On inhibition/disinhibition in developmental psychopathology: views from cognitive and personality psychology and a working inhibition taxonomy. Psychological Bulletin, 126, 220-246.

Oberauer, K. (2001). Removing irrelevant information from working memory: a cognitive aging study with the modified Sternberg task. Journal of Experimental Psychology. Learning, Memory, and Cognition, 27, 948-957.

Oberauer, K. (2005a). Binding and inhibition in working memory: individual and age differences in short-term recognition. Journal of Experimental Psychology. General, 134, 368-387.

Oberauer, K. (2005b). Control of the contents of working memory-a comparison of two paradigms and two age groups. Journal of Experimental Psychology. Learning, Memory, and Cognition, 31, 714-728.

Palladino, P., Mammarella, N., \& Vecchi, T. (2003). Modality-specific effects in inhibitory mechanisms: the interaction of peripheral and central components in working memory. Brain and Cognition, 53, 263-267.

Pelli, D. G. (1997). The VideoToolbox software for visual psychophysics: transforming numbers into movies. Spatial Vision, 10, 437-442.

Plude, D. J., \& Doussard-Roosevelt, J. A. (1989). Aging, selective attention, and feature integration. Psychology and Aging, 4, 98105. doi:10.1037/0882-7974.4.1.98.

Robert, C., Borella, E., Fagot, D., Lecerf, T., \& Ribaupierre, A. (2009). Working memory and inhibitory control across the life 
span: intrusion errors in the reading span test. Memory \& Cognition, 37, 336-345.

Rush, B. K., Barch, D. M., \& Braver, T. S. (2006). Accounting for cognitive aging: context processing, inhibition or processing speed? Aging, Neuropsychology, and Cognition, 13, 588-610. doi:10.1080/13825580600680703.

Salthouse, T. A. (1991). Mediation of adult age differences in cognition by reductions in working memory and speed of processing. Psychological Science, 2, 179-183.

Salthouse, T. A. (1996). The processing-speed theory of adult age differences in cognition. Psychological Review, 103, 403428.

Schelstraete, M. A., \& Hupet, M. (2002). Cognitive aging and inhibitory efficiency in the Daneman and Carpenter's working memory task. Experimental Aging Research, 28, 269279.

Scialfa, C. T., Esau, S. P., \& Joffe, K. M. (1998). Age, targetdistractor similarity and visual search. Experimental Aging Research, 24, 337-358.

Stoltzfus, E. R., Hasher, L., Zacks, R. T., Ulivi, M., \& Goldstein, D. (1993). Investigations of inhibition and interference in younger and older adults. Journal of Gerontology, 48, P179-P188.
Sullivan, M. P., \& Faust, M. E. (1993). Evidence for identity inhibition during selective attention in old adults. Psychology and Aging, 8, 589-598.

Tipper, S. P. (1985). The negative priming effect: inhibitory priming by ignored objects. The Quarterly Journal of Experimental Psychology, 37A, 571-590.

Toothaker, L. E. (1993). Multiple comparison procedures. Newbury Park: Sage.

Unsworth, N., \& Engle, R. W. (2007). The nature of individual differences in working memory capacity: active maintenance in primary memory and controlled search from secondary memory. Psychological Review, 114, 104-132.

Verhaeghen, P., \& De Meersman, L. (1998). Aging and the Stroop effect: a meta-analysis. Psychology and Aging, 13, 120-126. doi:10.1037/0882-7974.13.1.120.

Wechsler, D. (1981). WAIS-R: Wechsler adult intelligence scale (Revth ed.). New York: Psychological Corp.

West, R. L. (1996). An application of prefrontal cortex function theory to cognitive aging. Psychological Bulletin, 120, 272-292.

Wilson, S. P., \& Kipp, K. (1998). The development of efficient inhibition: evidence from directed-forgetting tasks. Developmental Review, 18, 86-123. 